\title{
Sken\&agraphie
}

SHínagaphiz Coulisses des arts du spectacle et des scènes

émergentes

5 | 2018

Juste la fin du monde, de Lagarce à Dolan

\section{Lagarce, d'une langue à l'autre : Entretiens avec Paolo Bellomo (italien), Marcel Kalunga (kiswahili) et Alina Kornienko (russe)}

Questionnaire proposé par Alexis Leprince

\section{Alexis Leprince}

\section{(2) OpenEdition}

\section{Journals}

Édition électronique

URL : http://journals.openedition.org/skenegraphie/1542

DOI : 10.4000/skenegraphie.1542

ISSN : 2553-1875

Éditeur

Presses universitaires de Franche-Comté

Édition imprimée

Date de publication : 1 janvier 2018

Pagination : 193-208

ISBN : 978-2-84867-5609-8

ISSN : $1150-594 \mathrm{X}$

Référence électronique

Alexis Leprince, «Lagarce, d'une langue à l'autre : Entretiens avec Paolo Bellomo (italien), Marcel Kalunga (kiswahili) et Alina Kornienko (russe) », Sken\&agraphie [En ligne], 5 | 2018, mis en ligne le 01 janvier 2019, consulté le 30 avril 2019. URL : http://journals.openedition.org/skenegraphie/1542 DOI : 10.4000/skenegraphie.1542

Ce document a été généré automatiquement le 30 avril 2019

Presses universitaires de Franche-Comté 


\section{Lagarce, d'une langue à l'autre : Entretiens avec Paolo Bellomo (italien), Marcel Kalunga (kiswahili) et Alina Kornienko (russe)}

Questionnaire proposé par Alexis Leprince

Alexis Leprince

Du 18 au 20 octobre 2007, le CDN de Besançon accueillait, en partenariat avec le Nouveau Théâtre et le Centre Jacques-Petit, le colloque "Traduire Lagarce: langue, culture, imaginaire ", dans le cadre de l'ensemble des "Colloques année (...) Lagarce "1. Alors que la diffusion mondiale de l'ouvre lagarcienne s'est depuis poursuivie, nous avons soumis, dix ans plus tard, trois traducteurs à un questionnaire. Il donne aussi sa place à des extraits faisant voir, et comme entendre, leur travail.

\section{Traduire Lagarce en italien : Paolo Bellomo²}

\section{Quelle(s) Pièce(s) de Jean luc lagarce aVez-vous traduite(s) et dans Quel} CADRE ?

J'ai traduit Juste la fin du monde en italien pour une création de la compagnie de théâtre Nostos que j'ai fondée en 2014 avec le metteur en scène Paolo Taccardo (http:// www.compagnienostos.com/about).

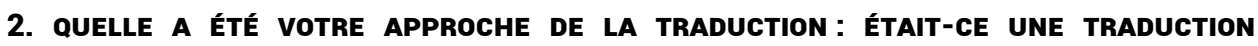
STRICTE ? UNE ADAPTATION ? AVEZ-VOUS EFFECTUÉ UNE TRANSPOSITION DES RÉFÉRENCES CULTURELLES SPÉCIfIQUES?

À l'origine de ma traduction de Juste la fin du monde se trouve une sorte de pulsion traductive. La langue extrêmement travaillée par l'auteur, à la fois chargée d'émotions et d'une précision rationnelle extraordinaire, a fait naître en moi le désir d'imposer la langue italienne à Lagarce et Lagarce à la langue italienne afin de voir «ce que ça donne ». Tout en me tenant extrêmement près du texte (un souci proche du littéralisme), j'ai traduit contre une certaine interprétation de l'œuvre: dans cette 
province française, chaque prise de parole m'est apparue moins une recherche abstraite d'un juste sens, une reproduction de la fragmentation de la pensée, que la tentative décidée, de tous les personnages, d'imposer aux autres un discours proche de la manipulation, de la torture. Tout en n'ayant pas opté pour une modification systématique du référentiel de l'œuvre, face à un mot dont la polysémie pouvait avoir un sens abstrait et un sens concret, j'ai presque toujours arrêté mon choix sur ce dernier.

Très peu de références culturelles sont présentes dans ce texte qui évolue plutôt dans l'univers et dans le vocabulaire des relations familiales (facilement compréhensibles entre France et Italie). Le seul cas d'adaptation regarde la race d'un chien qu'Antoine évoque à la scène 1 pour parler de sa sœur Suzanne. J'ai traduit l'original « On dirait un épagneul» par «Sembra un cocker». Un choix dicté moins par une difficulté référentielle que par le caractère percutant des sons et du rythme.

3. La langue de lagarce est très particulière ; CoMment la Rendre dans UNe AUTRE LANGUE SELON VOTRE EXPÉRIENCE DE TRADUCTION?

Cela a été un des enjeux majeurs de mon travail. Profitant du fait que l'action se déroule dans une province, j'ai essayé de créer une oralité plausible d'un italien provincial fortement influencé par des structures non seulement françaises mais lagarciennes ; en d'autres termes, j'ai essayé de faire passer l'étrangeté de cette écriture comme un idiotisme. Ainsi, non seulement j'ai gardé le vouvoiement (qui perdure d'ailleurs dans l'italien de plusieurs provinces du Sud de l'Italie) mais aussi des répliques telles que celle de Suzanne « mi telefoni oggi e ti sarei immediatamente venuta incontro » où la syntaxe déjoue la logique de l'italien standard. La liberté de prendre de tels choix a été aussi liée au type de travail fait dans la direction d'acteur par le metteur en scène Paolo Taccardo, qui vise un ancrage profond de la parole aux corps des comédiens rendant ce type de tournures immédiatement compréhensibles (du point de vue de la relation à l'action, à l'espace, aux autres personnages) pour les spectateurs.

\section{QUelles sont les difficultés spécifiques aU texte de lagarce que vous aVez} RENCONTRÉES?

Ce sont celles qui m'ont poussé vers la traduction : le défi d'en garder la jouabilité. Je dirais qu'elles se situent dans l'oralité quelque part entre le rythme et l'adresse : chaque partie de phrase - même celles qui ne se terminent pas, qui se perdent ou qui virent soudainement - est adressée. La difficulté était celle de garder cette adresse, de ne pas faire de cette langue très travaillée une langue écrite, de ne pas céder à un certain lyrisme.

5. comment s'est fait le passage de la traduction au plateau? avez-vous EFFECTUÉ DE NOUVELLES TRADUCTIONS ? DES COUPES DANS LE TEXTE ?

Nous sommes toujours en train de travailler sur la mise en scène. Une première résidence de création au Teatro delle Forche de Massafra (Italie) m'a permis de revenir sur certains passages de la traduction. Il s'est agi d'interventions ponctuelles : je n'ai pas eu besoin de revenir massivement sur un extrait. Nous avons effectué des coupes dans le texte, moins au niveau des scènes qu'au niveau de certains monologues.

\section{POUVEZ CITER, EN FRANÇAIS ET DANS LA LANgUE TRADUITE, UN COURT EXTRAIT TRADUIT DONT VOUS ESTIMEZ QUE LA TRADUCTION EST RÉUSSIE ?}

Je vous en cite deux; l'incipit de la pièce, assez représentatif du parler de Louis et un passage où c'est Antoine qui prend la parole (première partie, scène 11). 
Traduction en italien : incipit

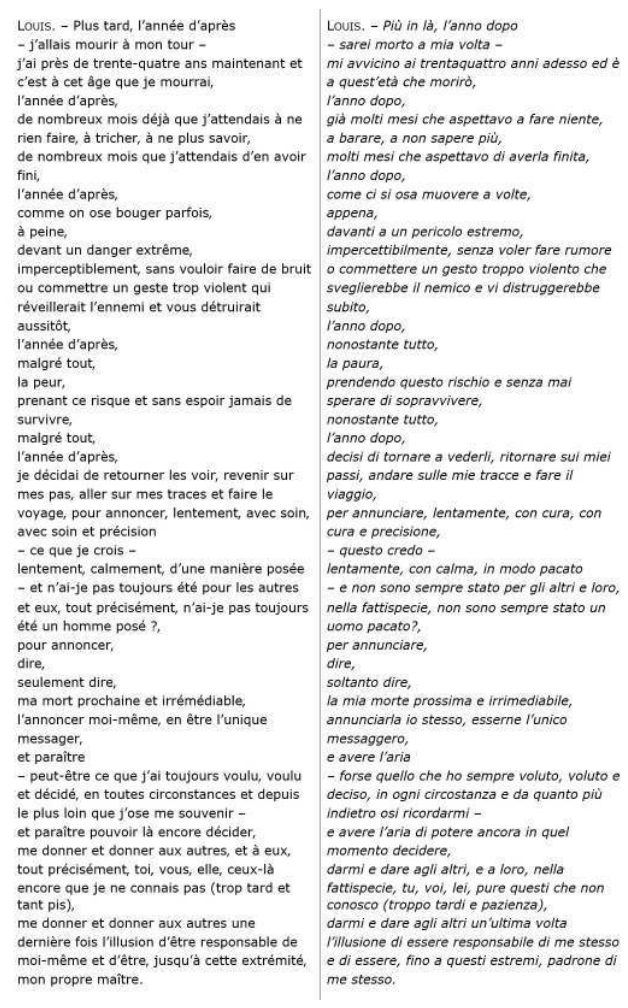

Traduction en italien : première partie, scène 11

\begin{tabular}{|c|c|}
\hline $\begin{array}{l}\text { ANTorne. - C'est cela, } \\
\text { c'est exactement cela, ce que je disais, } \\
\text { les histoires, } \\
\text { et après on se noie } \\
\text { et moi, } \\
\text { il faut que j'écoute et je ne saurai jamais ce } \\
\text { qui est vrai } \\
\text { et ce qui est faux, } \\
\text { la part du mensonge. } \\
\text { Tu es comme ca, } \\
\text { s'il y a bien une chose } \\
\text { (non, ce n'est pas la seule !), } \\
\text { s'ín y a bien une chose que je n'ai pas oubliée } \\
\text { en songeant à toi, } \\
\text { c'est tout cela, ces histoires pour rien, } \\
\text { des histoires, je ne comprends rien. }\end{array}$ & $\begin{array}{l}\text { ANToine, - E questo, } \\
\text { è esattamente questo, quello che dicevo, } \\
\text { le storie, } \\
\text { e poi uno affoga } \\
\text { e io, } \\
\text { devo ascoltare e non saprò mai cosa è vero } \\
\text { cosa è falso, } \\
\text { distsinguere le bugie. } \\
\text { Tu sei cosi, } \\
\text { se c'e proprio una cosa } \\
\text { (no, non è l'unica!), } \\
\text { se c'́ proprio una cosa che non ho } \\
\text { dimenticato quando penso a te, } \\
\text { è tutto questo, queste storie per niente, } \\
\text { storie, non capisco niente. }\end{array}$ \\
\hline 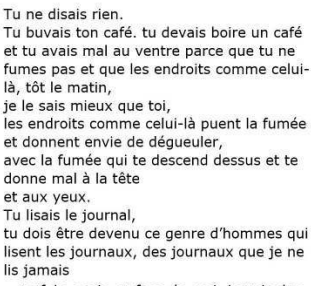 & 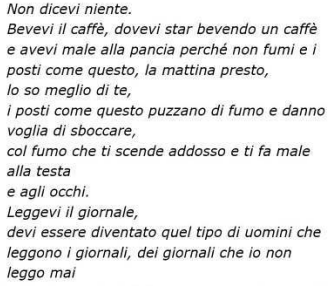 \\
\hline 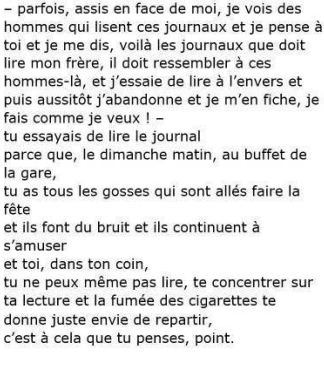 & $\begin{array}{l}\text { - a volte, seduti di fronte a me, vedo uomini } \\
\text { che leggono questi giornali e penso a te e mi } \\
\text { dico, eccoo igiornali che deve leggere mio } \\
\text { fratello, deve essomigliare a questi uomini, e } \\
\text { provo a leggere al contrario e poi } \\
\text { immediatamente lascio stare e me ne frego, } \\
\text { faccio come voglio! - } \\
\text { provavi a leggere il giornale } \\
\text { perché, la domenica mattina, al bar della } \\
\text { stazione, } \\
\text { ci sono tutti i ragazzetti che sono uscititi a far } \\
\text { serata } \\
\text { e fanno rumore e continuano a divertirsi } \\
\text { e tu, nel tuo angolino, } \\
\text { non puoi nemmeno leggere, concentrarti } \\
\text { sulla lettura e il fumo delle sigarette ti da } \\
\text { solo voglia di andare di nuovo via, } \\
\text { ea a questo che pensi, punto. }\end{array}$ \\
\hline
\end{tabular}




\section{Traduire Lagarce en kiswahili : Marcel Kalunga}

\section{Quelle(s) pièce(s) de Jean luc lagarce avez-vous traduite(s) et dans Quel CADRE ?}

J'ai traduit Les règles du savoir-vivre dans la société moderne. En plus de mes enseignements de langue française, j'enseignais aussi le kiswahili au sein de l'Alliance Française de Lubumbashi (R.D. du Congo). C'est l'un des responsables de l'Alliance qui m'avait demandé cette traduction. Celle-ci a été faite en kiswahili qui est, avec le hausa, l'une des plus grandes langues de l'Afrique noire.

Sur le plan géographique, le kiswahili est principalement parlé dans des pays situés à l'est et au centre du Continent : la Tanzanie, le Kenya, l'Ouganda, le Burundi, le Rwanda, la République Démocratique du Congo. Sa présence est également signalée en Somalie, au Malawi et au Mozambique.

Au niveau de la R.D. Congo, sur les 11 anciennes provinces administratives ${ }^{3}$, le kiswahili est utilisé dans les 5 provinces suivantes : Le Katanga, le Maniema, le Nord-Kivu, le SudKivu et la province Orientale. L'ensemble de la superficie de ces cinq provinces est supérieur à la superficie totale de la Tanzanie, du Kenya et de l'Ouganda réunis.

Le kiswahili est une langue bantu. Ce caractère bantu est confirmé par sa structure grammaticale qui est semblable à celle de beaucoup d'autres langues bantu. Son lexique, essentiellement constitué d'éléments proprement bantu, renferme aussi des éléments d'origines étrangères (langues asiatiques, l'arabe en l'occurrence, les langues européennes, anglais, français, portugais, etc.). Il existe aussi en kiswahili des mots d'origine bantu qu'il partage avec bien d'autres langues bantu. Et, comme beaucoup de langues du monde, le kiswahili comprend des dialectes dont le degré d'intercompréhension dépend de la distance qui sépare les différents parlers. Bref, la richesse du kiswahili est due à sa grande capacité d'assimiler des formes des langues étrangères qu'il ajoute aux siennes propres.

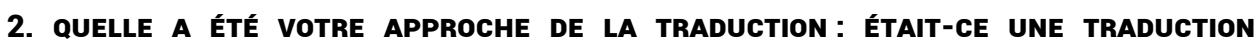
STRICTE ? UNE ADAPTATION ? AVEZ-VOUS EFFECTUÉ UNE TRANSPOSITION DES RÉFÉRENCES CULTURELLES SPÉCIfIQUES?

Il s'est agi, par endroits, de traductions strictes et, à d'autres endroits, d'adaptations. Certaines données culturelles n'ont pas été modifiées (par exemple, les noms des acteurs et des lieux). Des adaptions ont été portées à des prénoms (ceux qui apparaissent lors de la séquence du baptême) ainsi qu'à des noms de plantes et animaux.

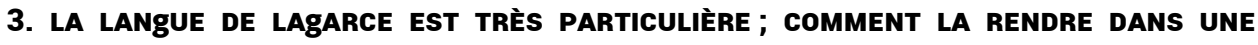
AUTRE LANGUE SELON VOTRE EXPÉRIENCE DE TRADUCTION ?

L'une des particularités du style de Lagarce réside dans l'abondance des répétitions : les va-et-vient: ce mouvement porte sur des mots, des locutions et même des temps verbaux.

À bien des endroits son style revêt un caractère oral : on sent et l'on voit quelqu'un qui parle d'une manière très libre, il se répète, interrompt son discours, laissant à l'auditeur le soin de compléter sa pensée. 
Ces aspects n'ont pas tellement nui à notre traduction étant donné que nous avons essayé de reproduire ce style.

\section{Quelles sont les difficultés spécifiques au texte de lagarce que vous aVez RENCONTRÉES?}

La première concerne les va-et-vient qui ne permettent pas de saisir l'intention de l'auteur. Par exemple :

Quand naissent des enfants jumeaux,

lorsqu'il y a des enfants jumeaux, si des enfants naissent jumeaux et que tous deux

restent vivants,

on doit,

on devra,

on doit faire connaitre l'ordre dans lequel ils sont nés, afin qu'on puisse établir quel est l'aîné, qui est l'aîné, lequel est l'aînét.

La seconde concerne des facteurs culturels, notamment ceux relatifs à l'organisation du baptême, des fiançailles, du mariage et du deuil.

D’une manière générale, les modifications relatives aux données culturelles portaient, les unes sur des titres honorifiques, les autres sur quelques noms propres (des personnes ou des plantes) et les autres encore sur des réalités inconnues dans l'aire d'emploi de la langue de traduction.

Voici, à titre d'exemples, comment nous avons traduit certains noms de fleurs :

- Lilas = Lilasi

- Chrysanthème $=$ Krizantemi

- Scabieuse = Skabiusi

- Reine Marguerite = Malkia Margerita

Voici d'autres cas :

- on danse la gigue $=$ wanacheza

- Grands tribuns $=$ waheshimwa wakubwa

- Duchesse = Mama Mheshimiwa

- Octave de Pâques = Siku nane baada ya Pasaka

- Lophophore $=$ Lofofori

Pour les traduire, nous avons parfois eu recours à un terme général (« on danse la gigue », traduit par «wanacheza » qui signifie simplement «Ils jouent / ils dansent » sans préciser la particularité de la gigue).

Il peut s'agir de syntagmes: «Duchesse » est traduit par «Mama mheshimiwa») qui, littéralement, veut dire « Femme respectable / vénérable / digne de respect ». " Grands Tribuns" devient "Waheshimiwa wakubwai » qui, littéralement, correspondrait à «Grandes personnalités respectables».

On peut aussi procéder par calques : «Octave de Pâques » devient «Siku nane baada ya Pasaka » qui signifie littéralement huit jours après Pâques. Il en est de même pour la fleur dite «Reine Marguerite» pour laquelle nous n'avons pris que des équivalents swahilis, soit «Malkia Margerita ».

Des mots tels que "Lilas ", "Scabieuse ", "Chrysanthème ", " lophophore " ont donné naissance à des formes swahilies forgées par nous selon ces quelques principes :

- en adaptant la graphie habituellement en usage en swahili; par exemple $\mathrm{ph}=\mathrm{f}$ (« lophophore $») ; \mathrm{chr}=\mathrm{kr}$ et th = $\mathrm{t}($ ( chrysanthème $) ; \mathrm{sc}=\mathrm{sk}($ ( Scabieuse $»)$;

- en dotant toutes les formes d'une voyelle finale en « $\mathrm{i} »$. 
Pour des facilités de prononciation, nous avons préféré «skabiusi » à «skabiesi » (« Scabieuse »).

L'un des objectifs majeurs de la traduction est de faciliter la compréhension du message de la langue 1 par le lecteur de la langue 2. Néanmoins, pour une personne qui n'a aucune idée de ce qu'est un "lophophore", par exemple, serait-elle plus avancée lorsqu'on lui présente le mot «lofofori», bien que sa prononciation se conforme aux habitudes de la langue de traduction?

\section{COMment s'est fait le passage de la traduction au plateau? aVez-vous EFFECTUÉ DE NOUVELLES TRADUCTIONS ? DES COUPES DANS LE TEXTE ?}

Je n'ai pas de précisions sur des représentations qui auraient été réalisées à partir de ma traduction. Celle-ci a été publiée dans le même volume que celle de Victor Hugo (Le Roi s'amuse) que j'ai également effectué. Cette dernière traduction a quant à elle été jouée à l'Alliance Française de Nairobi (Kenya).

\section{POUVEZ-VOUS CITER, EN FRANÇAIS ET DANS LA LANgUe TRADUITE, UN COURT EXTRAIT TRADUIT DONT VOUS ESTIMEZ QUE LA TRADUCTION EST RÉUSSIE ?}

Selon moi, une traduction est réussie lorsqu'elle arrive à rendre dans la langue d'arrivée (L2) :

- le maximum des formes linguistiques de la langue de départ (L1);

- le maximum du contenu de la langue L1;

- les aspects supra-linguistiques (esthétique, notamment) de la langue L1.

Ces trois conditions fondamentales nous semblent avoir été remplies dans l'extrait suivant, bien que nous soyons conscients que, à trois ou quatre endroits, il nous ait fallu forger de nouveaux mots.

Traduction en kiswahili

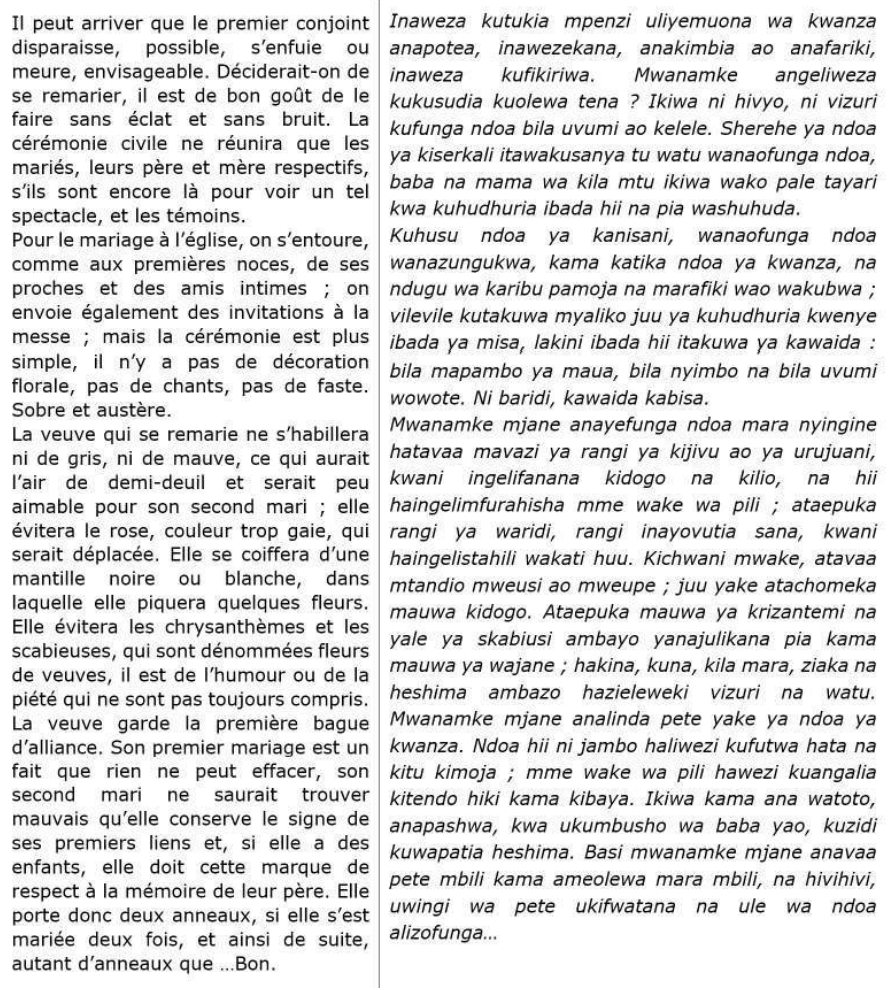




\section{Traduire Lagarce en russe : Alina Kornienko}

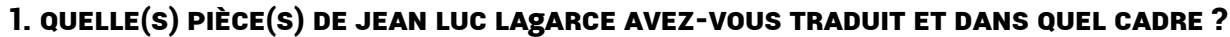

J'ai traduit en russe Derniers remords avant l'oubli afin qu'elle puisse être mise en scène en Russie. Cette traduction s'inscrit dans la suite du travail sur ma thèse intitulée Le théatre de Jean-Luc Lagarce: les infinis locataires de la parole. J'ai traduit pour ce travail de recherche des extraits des différentes pièces de Jean-Luc Lagarce comme J'étais dans ma maison et j'attendais que la pluie vienne, Les Règles du savoir-vivre dans la société moderne et Le Pays lointain.

Le corpus des pièces de Lagarce traduites en Russie entre 2007 et 2017 présente une sélection réduite à sept œuvres. Il n'y a jamais eu le projet de traduire l'intégralité des textes dramatiques de l'auteur mais plutôt des pièces précises, choisies pour des raisons spécifiques: la traduction dépendait d'initiatives en provenance soit des théâtres et compagnies russes, soit des projets de co-production avec la participation d'un metteur en scène français travaillant avec des acteurs russes.

Le choix de traduire - ou mettre en scène - les pièces de Jean-Luc Lagarce vient du fait qu'il est considéré, à l'étranger - et en Russie tout particulièrement -, comme un auteur dramatique français de premier plan, au même titre que Valère Novarina, Bernard Marie Koltès, Didier-Georges Gabily, ou Michel Vinaver, témoignant comme ces derniers d'un « retour au texte, souvent sous-entendu par l'ambition [...] d'inventer une langue nouvelle qui ne serait pas aliénée à la mise en scène à venir ${ }^{5} »$. On peut noter également que la pièce J'étais dans ma maison et j'attendais que la pluie vienne a obtenu une réception particulière en Russie. C'est la pièce de Jean-Luc Lagarce qui y est la plus mise en scène, notamment parce qu'elle apparait comme héritière de l'œuvre d'Anton Tchekhov. Les Règles du savoir-vivre dans la société moderne est également un texte souvent traduit et mis en scène à l'étranger, car il correspond au goût général du spectateur contemporain pour l'écriture et de la culture française. Le texte est souvent apprécié pour sa satire de l'organisation de la société, et le comique, ainsi que la forme monologique, ouvrent une possibilité d'interaction avec le public au cours du spectacle. Les récentes mises en scène des œuvres de Lagarce en Russie - même si leur nombre reste toujours relativement peu nombreux - montrent l'intérêt constant du public russe envers l'art, la littérature et le théâtre européen et plus précisément français.

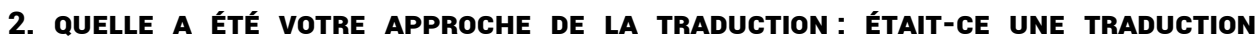
STRICTE ? UNE ADAPTATION ? AVEZ-VOUS EFFECTUÉ UNE TRANSPOSITION DES RÉFÉRENCES CULTURELLES SPÉCIfIQUES?

Paul Ricœur présentait la traduction comme un acte synchroniquement empirique et transcendantal. Malgré l'existence d'un très grand nombre de théories de traduction, il est évident que la traduction d'une œuvre dramatique en général, et du théâtre de texte en particulier, doit transmettre et transcrire le rythme et la poétique au sein desquels se cache la théâtralité du langage tel que définie par Henri Meschonnic. La traduction de l'œuvre dramatique est donc destinée à transmettre dans son intégralité et dans sa vivacité la tradition orale du langage originaire. Pour ce type de traduction littéraire, la transposition du sens passe, par conséquent, au second plan. Meschonnic postulait que la traduction du texte dramatique, qu'il considérait être une forme poétique, doit viser 
le rythme et non le sens des mots. C'est dans cette optique que j'ai travaillé ma traduction de Derniers remords avant l'oubli.

\section{QUELles SONT LeS difficultés spécifiques aU texte de lagarce QUe vous aVez RENCONTRÉES ?}

Les œuvres dramatiques de Lagarce peuvent sembler, de prime abord, une matière simple et claire pour le travail de traducteur. C'est dans cette simplicité et pureté illusoires de son langage que réside le plus grand piège pour les traducteurs, et les traductions existantes aujourd'hui mettent au jour les tournures problématiques, les constructions difficiles à transposer dans une langue syntaxiquement très différente. La structure répétitive et cacophonique du dialogue déconstruit mis en œuvre par Lagarce, la maladresse délibérée d'une énonciation qui se cherche, sont des particularités poétique et rythmique significativement épineuses pour la transcription. La fluidité d'un discours discontinu propre à l'écriture dramatique et littéraire est ainsi souvent perdue dans toutes les traductions de ses pièces en russe, ce qui appauvrit sa valeur dramatique et littéraire et rend sa réception difficile pour le spectateur russophone.

La plus grande faiblesse des traductions de Lagarce en russe consiste en une tentative de transmission du sens qui ignore souvent la sonorité particulière de ses œuvres. Certains traducteurs n'ont pas respecté la ponctuation spécifique introduite par Lagarce et sa mise en page qui participe au développement du rythme particulier du texte dramatique, et ont souvent ignoré le phénomène de répétition, éliminant parfois des passages entiers de séquences répétitives au sein d'une réplique. Les difficultés des traductions des œuvres de Lagarce en russe sont parfois lisibles dans certains cas dès le titre de la pièce : par exemple, sa dernière pièce devient littéralement en russe Dans le pays lointain, ce qui apporte un autre sens à l'œuvre construite autour du concept métaphorique de pays lointain. Mettant ce concept dans le titre de sa pièce, Lagarce n'a pas donné la signification directionnelle qu'a ajoutée la traductrice et qui a pour effet de réduire ce concept au spectre de ses significations spatiales.

La différence des systèmes langagiers du français et du russe rend significativement difficile la transposition des multiples hésitations langagières et lexicales qui forment la poétique lagarcienne. Ces hésitations, qui s'organisent à partir des formes du subjonctif et du conditionnel - ainsi par exemple que l'usage du pronom impersonnel « on » - ne peuvent être rendues qu'imparfaitement en langue russe. En effet, le russe ne contient que trois temps verbaux - un présent, un passé et un futur. Et précisons également qu'en russe le mode subjonctif n'existe pas. L'imparfait, que Lagarce utilise beaucoup, perd également, lors du passage à la langue russe, sa capacité à présenter un procès dans son déroulement. Pour cette raison, tout le texte de J'étais dans ma maison et j'attendais que la pluie vienne perd en russe d'une manière significative cette poéticité créée par l'usage de l'imparfait. En outre, les changements de temps verbaux du passé opérés par Lagarce se perdent également lors de la traduction en langue russe. Toutes les nuances et les différences de sens exprimées par Lagarce en français avec les temps et les modes verbaux sont rendues en russe par l'ajout de participes et d'adverbes. Le traducteur doit, par conséquent, non seulement garder le sens original de ces hésitations, mais également les exprimer dans le paradigme sonore auctorial d'une rhapsodie, ce qui constitue également une difficulté en raison de la sonorité différente de la langue russe. Dans la lignée de Meschonnic, le traducteur des œuvres dramatiques de Lagarce en langue russe doit s'efforcer de réinventer le rythme de l'auteur dans la 
langue source afin que ce rythme résonne aussi fort et de manière aussi familière pour le spectateur russe. Il doit, conformément à l'invention dramatique et littéraire de Lagarce, s'identifier aux membres de la fiction dramatique dans leur langage quotidien scrupuleusement retranscrit. Le spectateur doit également sentir l'atmosphère close et étouffante d'une absence de compréhension et de communication vécue par les personnages.

Sur le plan méthodologique, la traduction de l'œuvre littéraire théâtrale doit se produire selon trois axes : celui de la traduction du sens, celui de la traduction du rythme et celui de la conservation de la syntaxe et de la ponctuation de l'auteur. La problématique générale de la traduction qui consiste à choisir entre une imitation et une préservation doit, selon les expérimentations, toujours dépendre du système langagier de la langue sur laquelle la traduction est effectuée.

\section{4. comment s'est fait le passage de la traduction au plateau? avez-vous EFFECTUÉ DE NOUVELLES TRADUCTIONS ? DES COUPES DANS LE TEXTE ?}

La pièce Derniers remords avant l'oubli n'a pas encore été mise en scène en Russie.

\section{POUVEZ-VOUS CITER, EN FRANÇAIS ET DANS LA LANgUe TRADUITE, UN COURT EXTRAIT TRADUIT DONT VOUS ESTIMEZ QUE LA TRADUCTION EST RÉUSSIE ?}

J'ai choisi le monologue de Lise dans Derniers remords avant l'oubli. Il démontre la poétique dramatique et littéraire de Jean-Luc Lagarce. Ce monologue comprend également une signifiance particulière avec un code socioculturel qui n'est pas immédiatement accessible pour un lecteur / spectateur étranger et que le traducteur doit néanmoins réussir à transmettre. Je pense avoir finalement réussi à l'adapter complètement à la sonorité de la langue russe tout en le rendant accessible d'un point de vue culturel.

\section{Traduction en russe}

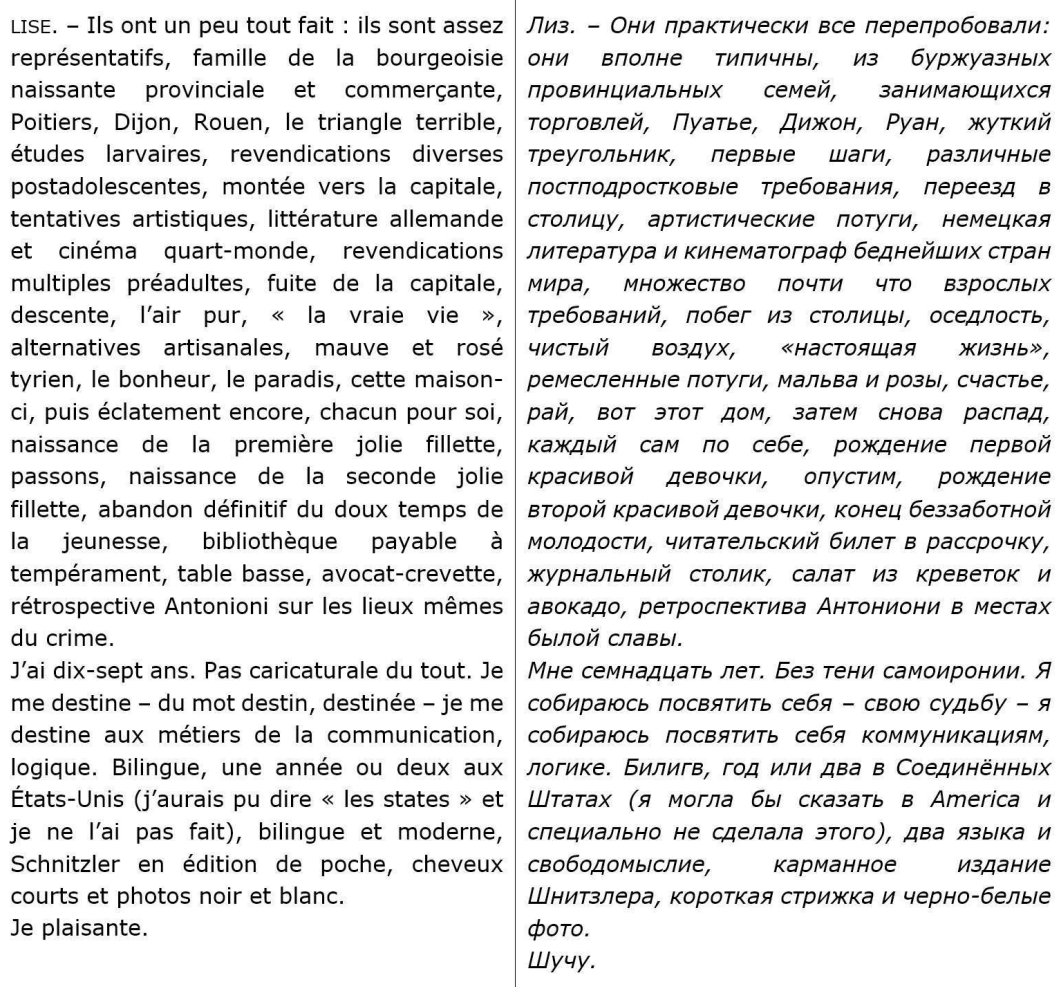

J'ai dix-sept ans. Pas caricaturale du tout. Je me destine - du mot destin, destinée - je me destine aux métiers de la communication, logique. Bilingue, une année ou deux aux États-Unis (j'aurais pu dire « les states » et je ne l'ai pas fait), bilingue et moderne, Schnitzler en édition de poche, cheveux courts et photos noir et blanc. Je plaisante.

Лиз. - Они практически все перепробовали: они вполне типичны, из буржуазных провинциальных семей, занимающихся торговлей, Пуатье, Дижон, Руан, жуткий треугольник, первые шаги, различные постподростковые требования, переезд в столицу, артистические потуги, немецкая литература и кинематограф беднейших стран мира, множество почти что взрослых требований, побег из столицы, оседлость, чистый воздух, «настоящая жизнь», ремесленные потуги, мальва и розы, счастье, рай, вот этот дом, затем снова распад, каждый сам по себе, рождение первой красивой девочки, опустим, рождение второй красивой девочки, конец беззаботной молодости, читательский билет в рассрочку, журнальный столик, салат из креветок и авокадо, ретроспектива Антониони в местах былой славы.

Мне семнадцать лет. Без тени самоиронии. Я собираюсь посвятить себя - свою судьбу - я собираюсь посвятить себя коммуникациям, логике. Билигв, год или два в Соединённых Штатах (я могла бы сказать в America и специально не сделала этого), два языка и свободомыслие, карманное издание Шнитзлера, короткая стрижка и черно-белые фото.

Шучу. 


\section{NOTES}

1. Les actes ont paru dans Traduire Lagarce - Langue, culture, imaginaire, Colloques Année (...) Lagarce, vol. III - Colloque de Besançon, Besançon, Les Solitaires intempestifs, 2008.

2. Nous remercions Teresa Solis qui a pris la charge des échanges avec Paolo Bellomo.

3. Après les dernières réformes administratives, le pays a été divisé en 26 provinces!

4. Jean-Luc LAGARCE, Les règles du savoir-vivre dans la société moderne, Théâtre complet, tome IV, Besançon, Les Solitaires intempestifs, 2010, p. 14.

5. Dominique VIART, Bruno VERCIER, La Littérature française au présent, Paris, Bordas, 2005.

\section{INDEX}

Mots-clés : Jean-Luc Lagarce, Juste la fin du monde, Le Pays lointain, Paolo Bellomo, Marcel Kalunga, Alina Kornienko, théâtre et traduction

\section{AUTEUR}

\section{ALEXIS LEPRINCE}

Alexis Leprince est doctorant boursier de la MSHE Claude Nicolas Ledoux à l'Université de Besançon Franche Comté où il poursuit une recherche en génétique sur l'œuvre théâtrale du dramaturge Jean-Luc Lagarce. Également praticien, il est le collaborateur régulier de la compagnie Artépo, en tant qu'assistant à la mise en scène et dramaturge. 